\title{
Study on cage slip characteristics of hybrid ceramic cylindrical roller bearing
}

\author{
Wenhu Zhang ${ }^{1, a}$, Sier Deng ${ }^{2,3, b}$, Guoding Chen ${ }^{1, c}$, Xue Sun ${ }^{1, d}$
}

1.School of Mechatronics Engineering, Northwestern Polytechnical University, Xi'an 710071, China

2. School of Mechatronics Engineering, Henan University of Science and Technology, Luoyang 471003, China
3.Collaborative Innovation Center of Major Machine Manufacturing in Liaoning, Dalian 116024 , China

a.526916105@qq.com, b.dse@haust.edu.cn, c.gdchen@nwpu.edu.cn, d.64354106@qq.com

\begin{abstract}
KeyWords: Slip ratio; GSTIFF; Dynamics; Hybrid ceramic cylindrical roller bearing
Abstract:In order to investigate the cage slip ratio, the dynamic differential equations of high-speed cylindrical roller bearing were built and solved by GSTIFF (Gear Stiff) integer algorithm. Cage slip ratio in hybrid ceramic cylindrical roller bearing and steel cylindrical roller bearing has been studied, and then the bearing test rig used for measuring cage slip ratio was presented. The results have shown that: Under the same working condition, cage slip ratio of hybrid ceramic cylindrical roller bearing is less than that of steel cylindrical roller bearing, it indicates that roller's material makes a great influence on cage slip ratio and hybrid ceramic cylindrical roller bearing is better to work at high-speed and light-load.
\end{abstract}

\section{Introduction}

The dynamic characteristics of cage make a great impact on the integrative performance of high-speed cylindrical roller bearings in main shaft of aero-engine. An excessive cage slip ratio exacerbates the friction between cage and guide ring and cage's unsteadily and breakdown. As a significant index of dynamic characteristics, cage slip ratio has been attracted much focus of researchers.

With the increase of rotating speed of main shaft in aero-engine, cylindrical roller bearing needs to bear much higher speed. Consequently, the issues due to the high-speed are emerged suddenly and can't be neglected. Kingsbury ${ }^{[1]}$ firstly developed a cage whirl model to study the whirl mechanism of cage and validated it by means of experiments, but he didn't analyzed the influential factors of cage whirl. Gupta ${ }^{[2,3]}$ derived the kinetic relationship of component parts and dynamic differential equations, and investigated the effect of friction coefficient and pocket clearance on the cage whirl radius and cage mass center orbit. Meek ${ }^{[4]}$ studied the forces acted on the cage and the wear of cage, however, he didn't analyze the cage dynamic characteristics. Niranjian Ghaisas ${ }^{[5]}$ introduced a model with six freedoms to study the effect of bearing clearance on cage dynamic characteristics. Jinlin $\mathrm{Hu}$, Guochang Lin, Linfeng Wu, Chengtie Zhang, etc. ${ }^{[6-10]}$ studied the dynamic characteristics of cage, but their studies are all based on quasi-static or quasi-dynamics, the theoretical basis remains relatively undeveloped. Zhihua Zhang ${ }^{[11]}$ developed the instantaneous kinetic equations of cage, and he adapted Newton-Raphson and RK45 to study the dynamic characteristic of cage and analyzed the effect of guide clearance and pocket clearance on the stability of cage. Haisheng Yang ${ }^{[12]}$ made the cage flexibility, and based on dynamics of bearing to investigate the influence of working conditions and structural parameters on the dynamic characteristics of cage. Sier Deng, Jinfang $\mathrm{Gu}{ }^{[13]}$ built the dynamic model of cylindrical roller bearing, analyzed cage guidance, the ratio of pocket clearance, and working conditions on cage's cage slip ratio.

In order to investigate the cage slip ratio, the dynamic differential equations of high-speed cylindrical roller bearing were built and solved by GSTIFF (Gear Stiff) integer algorithm. Cage slip 
ratio of hybrid ceramic cylindrical roller bearing and steel cylindrical roller bearing has been studied and then validated by experiments.

\section{Dynamics model of cylindrical roller bearings}

In order to exactly describe the state of motion, interaction forces and movement relationships of each bearing component, it is necessary to define the following five coordinate systems in Fig. 1.

(1) Inertial coordinate system $\{O ; X, Y, Z\}$ is fixed in space, $X$ axis coincides with rotating axis of bearing, $Y Z$ plane parallels to radial plane through bearing center.

(2) Coordinate system of roller mass center $\left\{o_{\mathrm{r}}, x_{\mathrm{r}}, y_{\mathrm{r}}, z_{\mathrm{r}}\right\}, o_{\mathrm{r}}$ coincides with roller's mass center, $y_{\mathrm{r}}$ axis is along radial direction of bearing, $z_{\mathrm{r}}$ axis is along circumferential direction of bearing. The coordinate system moves but doesn't spin with roller's mass center, each roller has its own local coordinate system.

(3) Coordinate system of cage's mass center $\left\{o_{c} ; x_{\mathrm{c}}, y_{\mathrm{c}}, z_{\mathrm{c}}\right\}, x_{\mathrm{c}}$ axis coincides with rotating axis of cage, $y_{\mathrm{c}} z_{\mathrm{c}}$ plane parallels to radial plane through cage center, $o_{\mathrm{c}}$ coincides with geometric center of cage, $\left\{o_{\mathrm{c}} ; x_{\mathrm{c}}, y_{\mathrm{c}}, z_{\mathrm{c}}\right\}$ moves and spins with cage.

(4) Coordinate system of inner ring mass center $\left\{o_{\mathrm{i}} ; x_{\mathrm{i}}, y_{\mathrm{i}}, z_{\mathrm{i}}\right\}, \quad x_{\mathrm{i}}$ axis is along with rotating axis of inner ring, $y_{\mathrm{i}} z_{\mathrm{i}}$ plane parallels with radial plane through inner ring mass center, $o_{\mathrm{i}}$ coincides with geometric center of inner ring, $\left\{o_{\mathrm{i}} ; x_{\mathrm{i}}, y_{\mathrm{i}}, z_{\mathrm{i}}\right\}$ moves and spins with inner ring.

(5) Coordinate system of cage pocket center $\left\{o_{\mathrm{p}}, x_{\mathrm{p}}, y_{\mathrm{p}}, z_{\mathrm{p}}\right\}, o_{\mathrm{p}}$ coincides with geometric center of cage pocket, $y_{\mathrm{p}}$ axis is along radial direction of bearing, $z_{\mathrm{p}}$ axis is along circumferential direction of bearing. $\left\{o_{\mathrm{p}} ; x_{\mathrm{p}}, y_{\mathrm{p}}, z_{\mathrm{p}}\right\}$ moves and spins with cage, each cage pocket center has its own local coordinate system.

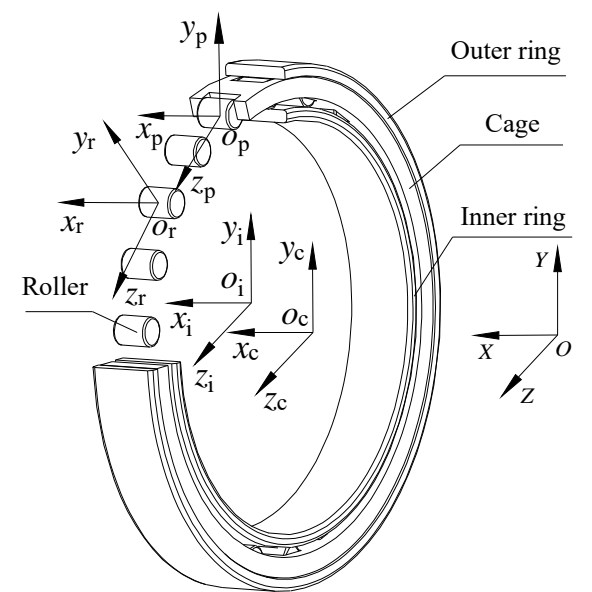

Fig.1. Coordinate systems of cylindrical roller bearing

Outer ring is static, inner ring rotates at constant speed $\omega$, inner ring only bears a radial force $F_{\mathrm{r}}$, and cage is guided by outer ring. The surfaces of bearing components are absolutely smooth and the mass center of each bearing component coincides with its centroid. The schematic diagram of cylindrical roller bearing is shown in Fig.2.

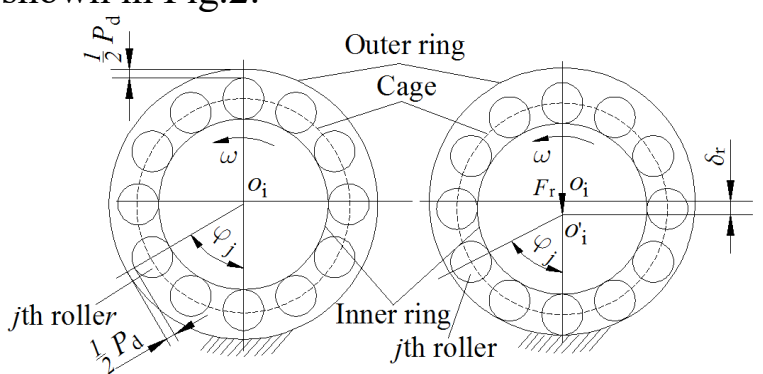

(a) un-loaded bearing (b)loaded bearing

Fig.2. Schematic diagram of cylindrical roller bearing 
In Fig.2, $\delta_{\mathrm{r}}$ is the radial displacement of inner ring; $P_{\mathrm{d}}$ is bearing radial clearance; $o_{\mathrm{i}}, o_{\mathrm{i}}^{\prime}$ are the mass center of inner ring of unloaded bearing and loaded bearing, respectively; $\varphi_{j}$ is azimuth angle of the $j$ th roller.

\section{Nonlinear dynamics differential equations of roller}

When bearing is working at high-speed, roller is simultaneously acted by combined forces of inner ring, outer ring and cage. The forces acting on the $j$ th roller are shown in Fig.3. The expressions of symbols in Fig.3 refer to Ref. [14].

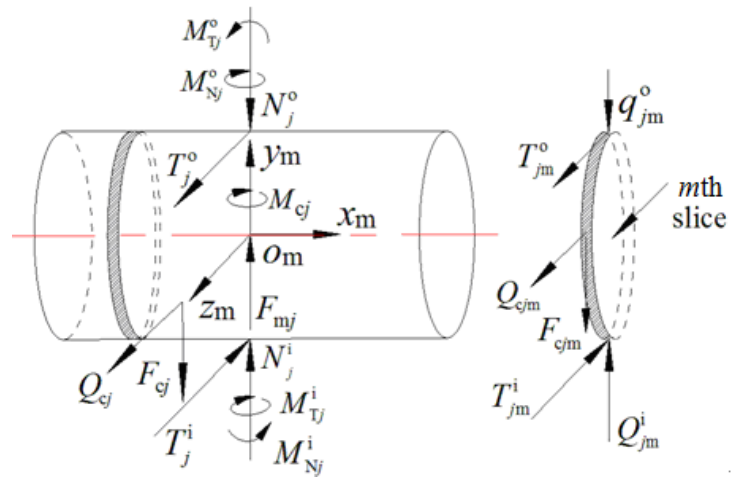

Fig.3 Schematic diagram of roller forces

In Fig.3, subscript $\{\mathrm{i}, \mathrm{o}\}$ represent inner raceway and outer raceway, respectively; $N_{j}^{\mathrm{i}}, N_{j}^{\mathrm{o}}$ are normal force between the $j$ th roller and raceways; $T_{j}^{\mathrm{i}}, T_{j}^{\mathrm{o}}$ are oil drag force between the $j$ th roller and raceways; $M_{\mathrm{N} j}^{\mathrm{i}}, M_{\mathrm{N} j}^{\mathrm{o}}$ are additional moment due to $N_{j}^{\mathrm{i}}$ and $N_{j}^{\mathrm{o}} ; M_{\mathrm{T} j}^{\mathrm{i}}, M_{\mathrm{T} j}^{\mathrm{o}}$ are additional moment due to $T_{j}^{\mathrm{i}}$ and $T_{j}^{\mathrm{o}} ; Q_{\mathrm{c} j}, F_{\mathrm{c} j}$ are normal force and tangential friction force between the $j$ th roller and cage's cross beam; $M_{\mathrm{c} j}$ is additional moment due to $F_{\mathrm{c} j} ; F_{\mathrm{m} j}$ is centrifugal force of the $j$ th roller; $q_{j \mathrm{~m}}^{\mathrm{i}}, q_{j \mathrm{~m}}^{\mathrm{o}}$ are contact forces between the $m$ th slice and raceways; $T_{j \mathrm{~m}}^{\mathrm{i}}, T_{j \mathrm{~m}}^{\mathrm{o}}$ are oil drag forces between the $m$ th slice and raceways; $Q_{\mathrm{cjm}}, F_{\mathrm{cjm}}$ are normal force and tangential friction force between the $m$ th slice and cage's cross beam.

The nonlinear dynamics differential equations of the $j$ th roller are shown as Eq.1.

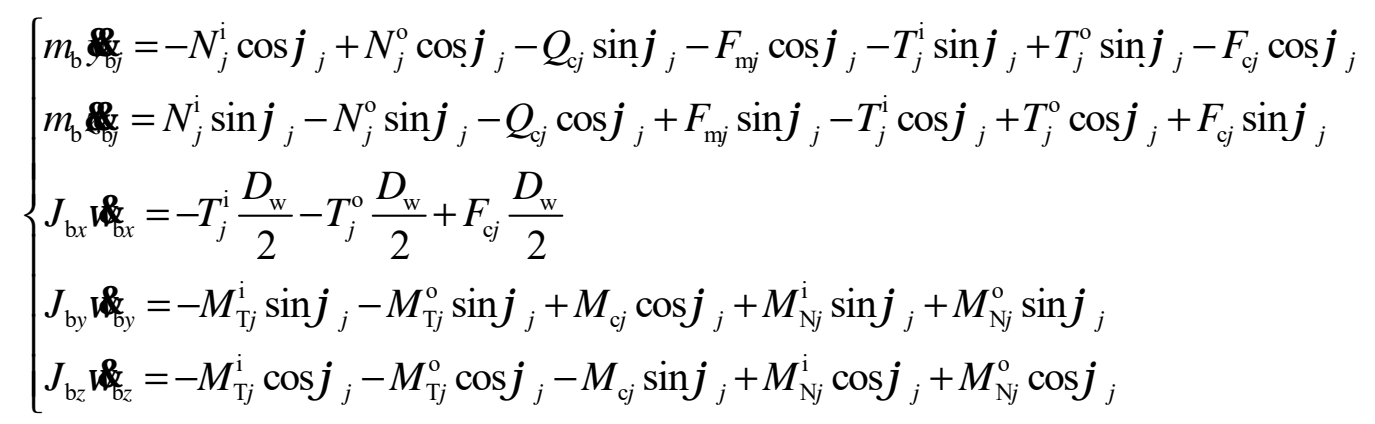

In Eq.1, $m_{\mathrm{b}}$ is roller mass; are displacement accelerations of the $j$ th roller's mass center in $\{O ; X, Y, Z\} ; J_{\mathrm{b} x}, J_{\mathrm{by}}, J_{\mathrm{bz}}$ are moments of inertia of roller in $\{O ; X, Y, Z\}$, respectively; $\alpha_{b x}, \omega_{b y}$, $\omega_{\delta}$ are angular accelerations of roller in $\{O ; X, Y, Z\} ; D_{\mathrm{w}}$ is roller diameter.

\section{Nonlinear dynamics differential equations of cage}

When bearing is working, cage is simultaneously acted by collision forces of rollers, guiding force of outer ring and combined resistance of oil/air mixture to cage's ends and surface, forces acting on cage are shown in Fig.4. The expressions of symbols in Fig. 4 refer to Ref. [14]. 


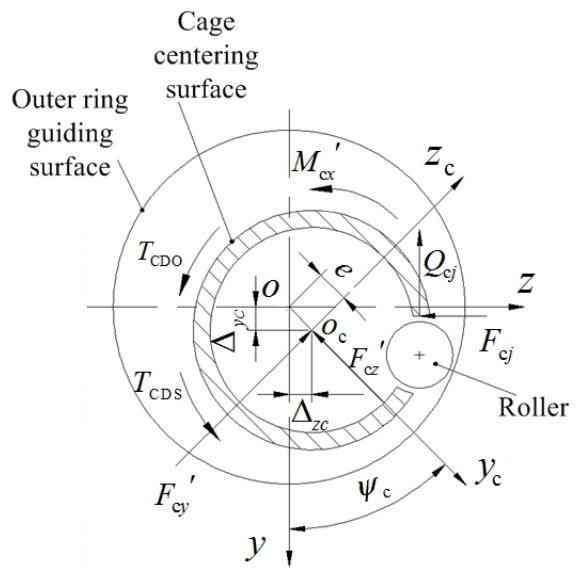

Fig.4 Schematic diagram of cage forces

In Fig.4, $\{o ; y, z\}$ is reference coordinate system of cage; $e$ is offset of cage center; $\Delta_{y c}, \Delta_{z c}$ are components of $e$ along $y$ axis and $z$ axis, respectively; $\Psi_{\mathrm{c}}$ is angle between $\left\{o_{\mathrm{c}} ; y_{\mathrm{c}}, z_{\mathrm{c}}\right\}$ and $\{o ; y, z\} ; F_{\mathrm{cy}}{ }^{\prime}, F_{\mathrm{c} z}{ }^{\prime}, M_{\mathrm{c} x}{ }^{\prime}$ are forces and moment caused by hydrodynamic action between cage centering surface and outer ring guiding surface, respectively; $T_{\mathrm{CDS}}, T_{\mathrm{CDO}}$ are the resistances of cage's end-surface and surface caused by oil/air mixture, respectively.

The nonlinear dynamics differential equations of cage are shown as Eq.2.

$$
\left\{\begin{array}{l}
m_{\mathrm{c}}=\sum_{j=1}^{R N}\left(Q_{\mathrm{c} j} \sin \varphi_{j}+F_{\mathrm{c} j} \cos \varphi_{j}\right)+F_{\mathrm{c} y}^{\prime} \cos \psi_{\mathrm{c}}+F_{\mathrm{c} z}^{\prime} \sin \psi_{\mathrm{c}}-G_{\mathrm{c}} \\
m_{\mathrm{c}}=\sum_{j=1}^{R N}\left(Q_{\mathrm{c} j} \cos \varphi_{j}-F_{\mathrm{c} j} \sin \varphi_{j}\right)+F_{\mathrm{c} y}^{\prime} \sin \psi_{\mathrm{c}}-F_{\mathrm{c} z}^{\prime} \cos \psi_{\mathrm{c}} \\
J_{\mathrm{c} x} \omega_{\mathrm{c} x}=\sum_{j=1}^{R N}\left(F_{\mathrm{c} j} \frac{D_{\mathrm{w}}}{2}\right)-M_{\mathrm{c} x}^{\prime}-T_{\mathrm{CDO}}-T_{\mathrm{CDS}} \\
J_{\mathrm{c} y} \omega_{\mathrm{c} y}=\sum_{j=1}^{R N}\left(-M_{\mathrm{c} j} \cos \varphi_{j}\right) \\
J_{\mathrm{cz}} \omega_{\mathrm{c} z}=\sum_{j=1}^{R N}\left(M_{\mathrm{c} j} \sin \varphi_{j}\right)
\end{array}\right.
$$

In Eq.2, $m_{\mathrm{c}}$ is cage mass; $G_{\mathrm{c}}$ is cage gravity; are displacement accelerations of cage's mass center in $\{O ; X, Y, Z\} ; J_{c x}, J_{c y}, J_{c z}$ are moments of inertia of cage in $\{O ; X, Y, Z\} ;$ o $\alpha_{c x}$, cosy , cox are angular accelerations of cage in $\{O ; X, Y, Z\} ; R N$ is the number of rollers.

Nonlinear dynamics differential equations of inner ring

The nonlinear dynamics differential equations of inner ring roller are shown as Eq.3. 


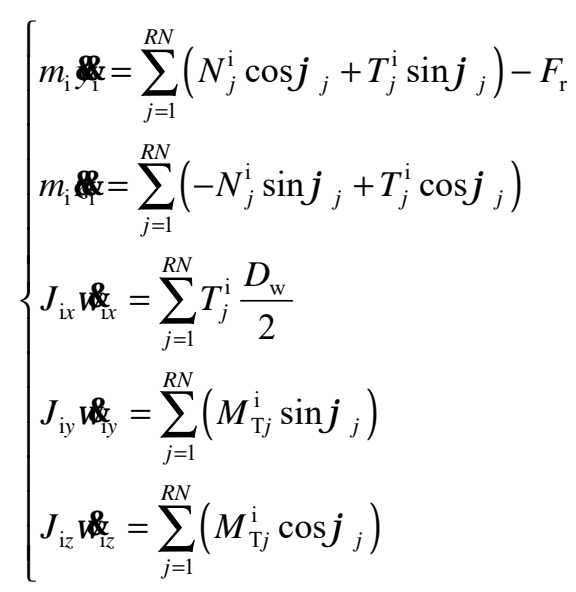

In Eq.3, $m_{\mathrm{i}}$ is mass of inner ring; are displacement accelerations of inner ring mass

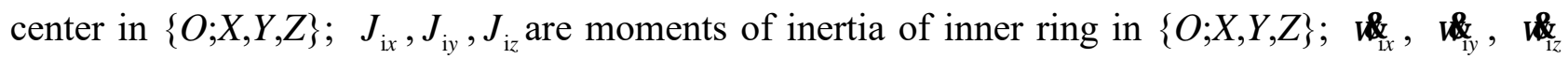
are angular accelerations of inner ring in $\{O ; X, Y, Z\}$.

\section{Solution procedure of dynamics differential equations}

GSTIFF $^{15]}$ integer algorithm with variable step was applied to solve dynamics differential equations of high-speed cylindrical roller bearing, solution procedure of dynamics differential equations was shown in Fig.5.

(1) Solution duration, initial step and convergence error of dynamics differential equations were set, firstly.

(2) According to initial estimated values of bearing component's position and motion constraint, initial conditions of dynamics differential equations, namely, relative position and motion vector of various components were obtained by quasi-static analysis.

(3) Given initial conditions of dynamics differential equations, forces and moments applied on bearing components were calculated according to the expressions in Ref.[14].

(4) The dynamics differential equations of bearing components were solved by GSTIFF, and then positions and motion vectors of bearing components were obtained.

(5) Verify whether the error obtained by the solution procedure of dynamics differential equations meets with the convergence error set in Step (1). If yes, then continue next solution procedure of step value after getting the outputs of motion parameters including displacement, velocity and accelerated speed of inner ring, cage and roller. If no, choose smaller step value and repeat Step (4) until solving error meets convergence error.

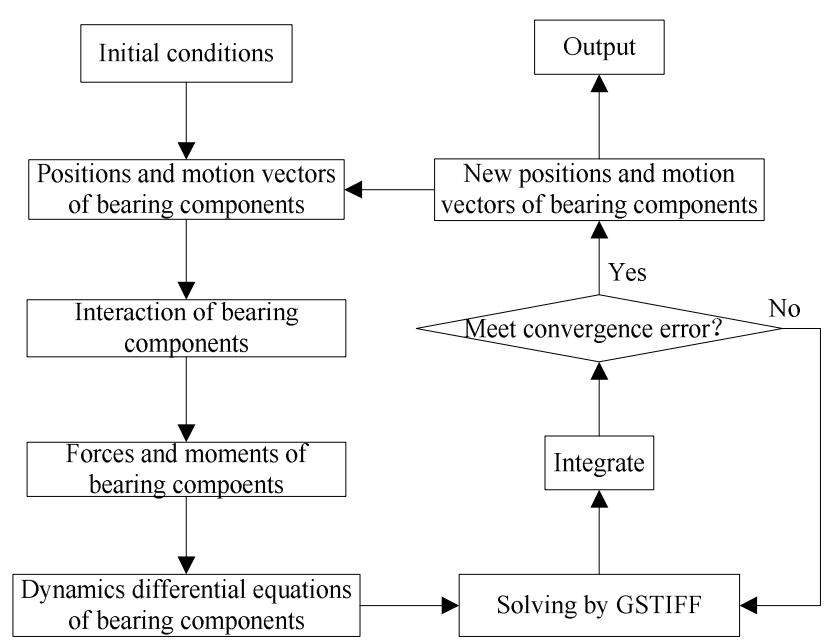

Fig.5 Solution procedure of dynamics differential equations 


\section{Comparison of Cage slip ratio between experiment and theoretical calculation}

The main bearing parameters were shown in Tab.1, and the parameters of rollers with S8Cr4Mo4V and Si3N4 were shown in Tab.2. Cage's dynamic performance test rig for aero-bearing ${ }^{[16]}$ was applied to validate the results of theoretical analysis and the test rig was shown in Fig.6.

Tab.1 Major parameters of bearing

\begin{tabular}{cc}
\hline Structural parameter & Value \\
\hline Bearing outer diameter $/ \mathrm{mm}$ & 140 \\
Bearing inner diameter $/ \mathrm{mm}$ & 110 \\
Bearing width $/ \mathrm{mm}$ & 19 \\
Roller diameter $/ \mathrm{mm}$ & 8 \\
Roller number & 34 \\
Roller length $/ \mathrm{mm}$ & 10 \\
Cage outer diameter $/ \mathrm{mm}$ & 132.4 \\
Cage inner diameter $/ \mathrm{mm}$ & 121.9 \\
\hline
\end{tabular}

Tab.2 Material parameters

\begin{tabular}{cccc}
\hline Material & Density $\left(\mathrm{g} / \mathrm{cm}^{3}\right)$ & Modulus of elasticity $\left(\mathrm{N} / \mathrm{mm}^{2}\right)$ & $\begin{array}{c}\text { Poisson's } \\
\text { ratio }\end{array}$ \\
\hline S8Cr4Mo4V & 8.21 & $2.07 \times 10^{6}$ & 0.3 \\
Si3N4 & 3.2 & $3.1 \times 10^{6}$ & 0.26 \\
\hline
\end{tabular}

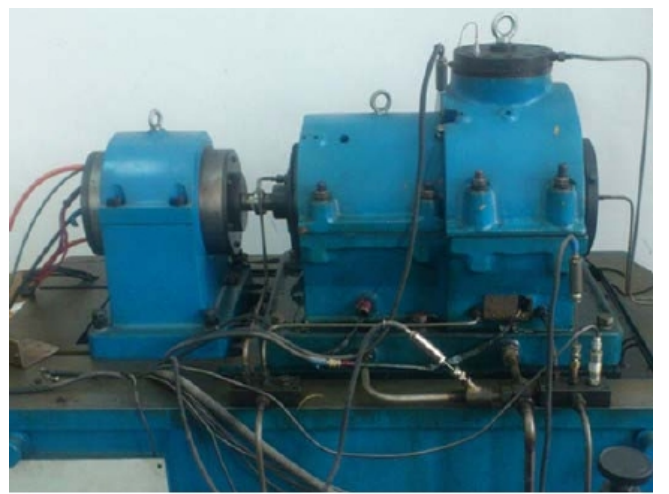

Fig.6 Cage dynamic performance test rig for aero-bearing

\section{Effect of rotating speed on cage slip ratio}

Cage slip ratio under 4000r/min, $5500 \mathrm{r} / \mathrm{min}, 7000 \mathrm{r} / \mathrm{min}, 8500 \mathrm{r} / \mathrm{min}$ and $10000 \mathrm{r} / \mathrm{min}$ were shown in Fig. 7 and Fig.8. With the rotating speed of inner ring increasing, cage slip ratio goes up and increases obviously at $7000 \mathrm{r} / \mathrm{min}$ in steel cylindrical roller bearing, but at $8500 \mathrm{r} / \mathrm{min}$ in hybrid ceramic cylindrical roller bearing. The centrifugal force was considered as the reason for the increase of cage slip ratio, with the rotating speed increasing, the centrifugal force of roller increased, which caused the inner ring can not drag the roller effectively. Because the density of ceramic material is smaller than that of $\mathrm{S} 8 \mathrm{Cr} 4 \mathrm{Mo} 4 \mathrm{~V}$, the centrifugal force of ceramic roller is less, and this is the reason why cage slip ratio of hybrid ceramic cylindrical roller bearing is less. 


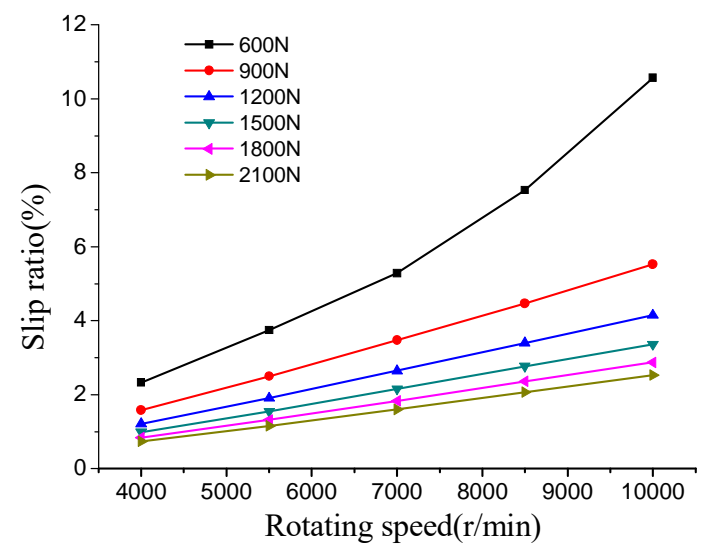

a) Theoretical calculation result

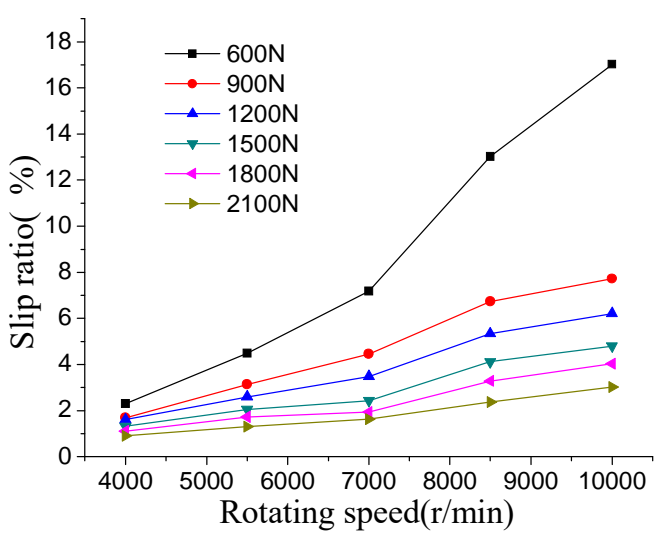

b) Experimental result

Fig.7 Trend of cage slip ratio with rotating speed in steel cylindrical roller bearing

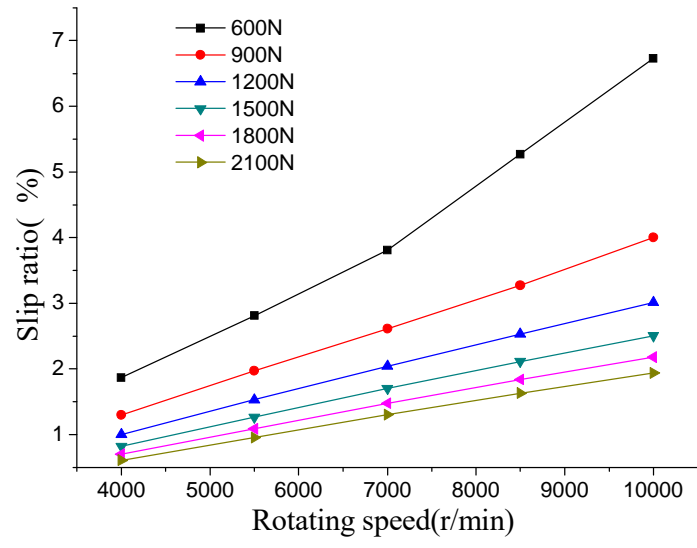

a) Theoretical calculation result

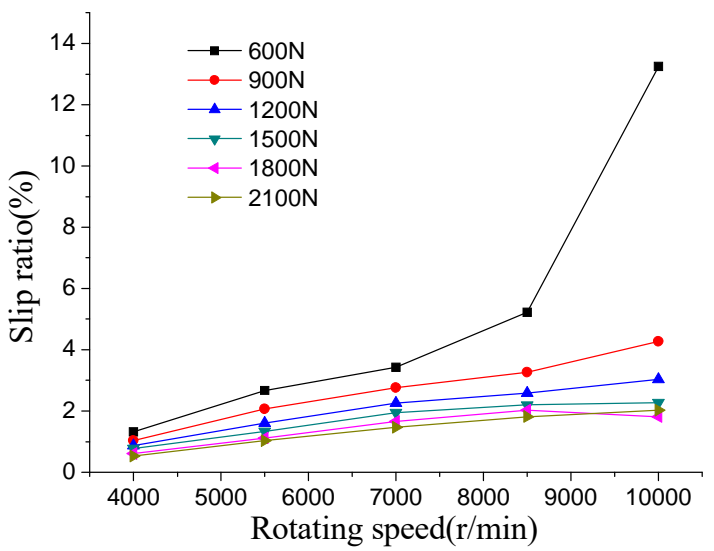

b) Experimental result

Fig. 8 Trend of cage slip ratio with rotating speed in hybrid ceramic cylindrical roller bearing

\section{Effect of radial load on cage slip ratio}

Theoretical calculation and experiment results in Fig.9 and Fig.10 show the trend of cage slip ratio under different radial load of $600 \mathrm{~N}, 900 \mathrm{~N}, 1200 \mathrm{~N}, 1500 \mathrm{~N}, 1800 \mathrm{~N}, 2100 \mathrm{~N}$. Cage slip ratio goes down gradually, and the downward trend varied gently after radial load exceeds $900 \mathrm{~N}$ in both bearings. The increasing radial force improved the drag between inner ring and roller, and then cage slip ratio decreased. However, due to the impact of lubricant, there was a limitation of drag force between inner ring and roller, after radial force is bigger than a certain value, the drag force between inner ring and roller changes less, and then cage slip ratio also changes less.

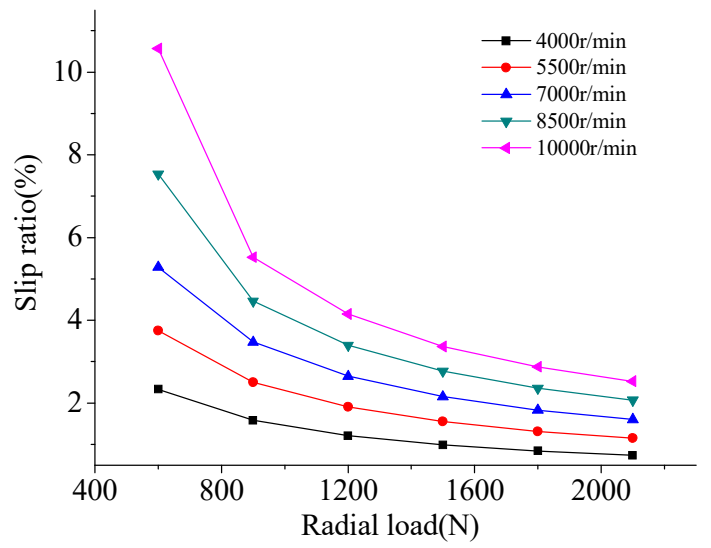

a) Theoretical calculation result

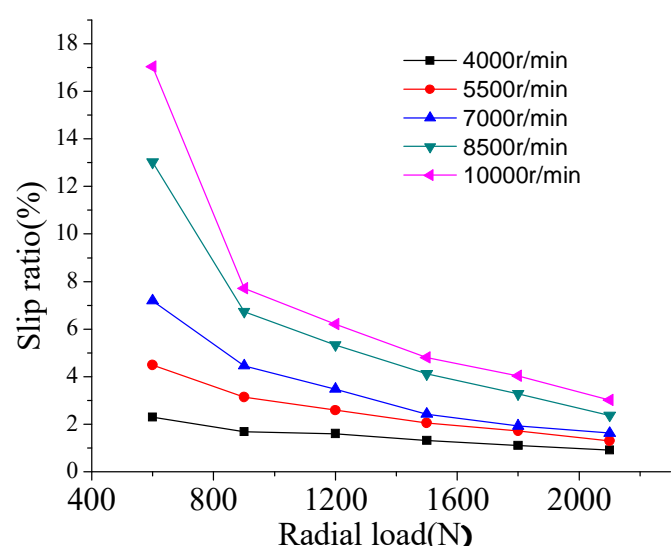

b) Experimental result

Fig.9 Trend of cage slip ratio changed with radial load in steel cylindrical roller bearing 


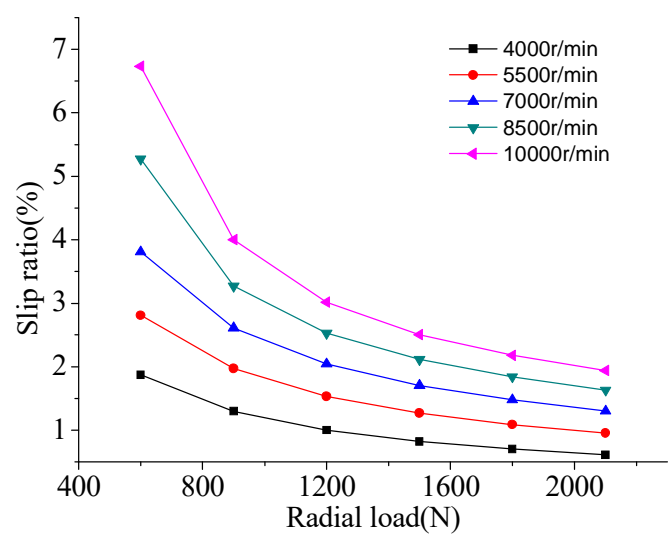

a) Theoretical calculation result

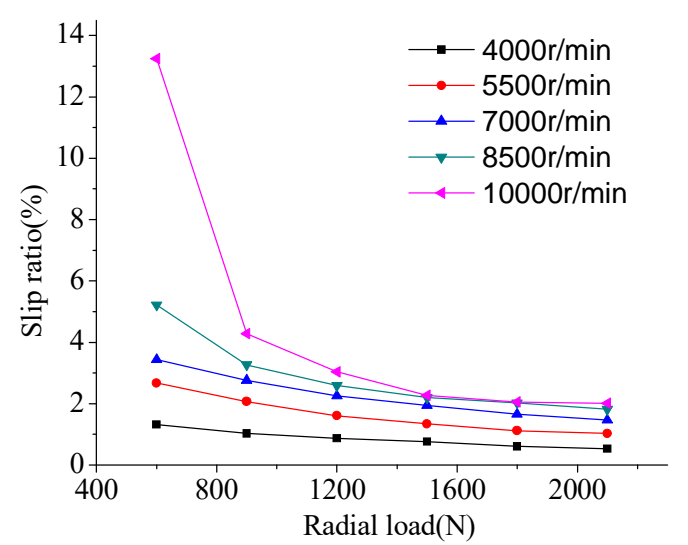

b) Experimental result

Fig. 10 Trend of cage slip ratio changed with radial load in hybrid cylindrical roller bearing

\section{Conclusions}

(1) With rotating speed increasing, due to the centrifugal force, cage slip ratio goes up and increases obviously at $7000 \mathrm{r} / \mathrm{min}$ in steel cylindrical roller bearing, but at $8500 \mathrm{r} / \mathrm{min}$ in hybrid cylindrical roller bearing.

(2) With radial load increasing, cage slip ratio goes down, and the downward trend varies gently after radial load exceeds $900 \mathrm{~N}$ in both types bearing which indicates that there exists a critical load to reduce the cage slip ratio.

(3) Under the same working condition, cage slip ratio of hybrid ceramic cylindrical roller bearing is less than that of steel cylindrical roller bearing, it indicates that roller's material makes a great influence on cage slip ratio and hybrid ceramic cylindrical roller bearing is better to work at high-speed and light-load.

\section{Acknowledgments}

This research is financially supported by National Natural Science Foundation of China(U1404514) and Collaborative Innovation Center of Major Machine Manufacturing in Liaoning.

\section{References}

[1] Kingsbury E, Walker R. Motions of an unstable retainer in an instrument ball bearing[J]. Journal of Tribology, Transaction of the ASME, 1994, 116(2): 202-208.

[2] Gupta P K. Dynamics of rolling element bearings, Parts I, II, III, and IV[J]. Journal of Lubrication Technology, 1979, 101(3): 293-326.

[3] Gupta P K. Dynamic loads and cage wear in high-speed rolling bearings[J]. Wear, 1991, 147(1): 119-134.

[4] Meeks C R. The dynamics of ball separators in ball bearings-Parts II: Results of optimization study[J]. Tribology Transactions, 1985, 28(3):288-295.

[5] Ghaisas N, Wassgren C R, Sadeghi F. Cage instabilities in cylindrical roller bearings[J]. Journal of Tribology, 2004, 126(4):681-689.

[6] Jinyu Hu, Jun Yu. Dynamic analysis of cage in high-speed cylindrical roller bearings[J]. Journal of Huazhong University of Science and Technology, 1989, 17(4):79-84[in Chinese].

[7] Guochang Lin. Quasi-static analysis of cylindrical roller bearings[J]. Journal of Aerospace Power, 1992, 7(1): 17-21[in Chinese].

[8] Linfeng Wu. Quasi-dynamics analysis of high-speed radial cylindrical roller bearings[J]. Journal of nanjing university of aeronautics \& astronautics, 1993,25(3):330-340[in Chinese].

[9] Tiecheng Zhang. Dynamic analysis of high-speed cylindrical roller bearings[J].Mechanical 
science and technology for aerospace engineering,1997,16(1):136-139[in Chinese].

[10] Li Cui. Dynamic characteristics analysis of high-speed cylindrical roller bearings in aero-engine[J]. Acta Aeronautica et Astronautica Sinica, 2008, 29(2): 492-498[in Chinese].

[11] Zhihua Zhang, Sier Deng. Dynamics analysis of cage in high-speed cylindrical roller bearings[J]. Henan University of Science and Technology, 2006,27(2):16-22[in Chinese].

[12] Haisheng Yang, Sier Deng. Flexible dynamic simulation on cage of aero-engine high speed cylindrical roller bearings[J].Bearing,2011,(2):7-11[in Chinese].

[13] Sier Deng, Jinfang Gu. Analysis on dynamic characteristics of cage in high-speed cylindrical roller bearing[J]. Journal of Aerospace Power, 2014,29(1):207-215[in Chinese].

[14] Sier Deng, Qunyi Jia, Jinxue Xue. Design principle of rolling bearings. Beijing: China Standard Press 2014.p. 225-237[in Chinese].

[15] Gear C W. Simultaneous Numerical solution of differential-algebraic equations[J]. IEEE Transactions on Circuit Theory, 1971,18(1):89-95..

[16] Sier Deng, Haisheng Yang, Chaoyang Sun. The test device for cage's dynamic performance of cylindrical roller bearings. China, CN202916093U[P], 2013[in Chinese]. 